\title{
Research Paper: Effect of Dynamic Neuromuscular Stabilization Breathing Exercises on Some Spirome- try Indices of Sedentary Students With Poor Posture
}

\author{
Nasser Mohammad Rahimi ${ }^{1}$ (D) , Reza Mahdavinejad ${ }^{1^{*}}$ (D), Seyyed Reza Attarzadeh Hosseini² (D) , Hossein Negahban ${ }^{3,4}$ \\ 1. Department of Sports Injuries and Corrective Exercises, Faculty of Sport Sciences, University of Isfahan, Isfahan, Iran. \\ 2. Department of Exercise Physiology, Faculty of Sports Sciences, Ferdowsi University of Mashhad, Mashhad, Iran. \\ 3. Department of Physical Therapy, School of Paramedical Sciences, Mashhad University of Medical Sciences, Mashhad, Iran. \\ 4. Orthopedic Research Center, Mashhad University of Medical Sciences, Mashhad, Iran.
}

\begin{tabular}{|l|l}
$\begin{array}{c}\text { Use your device to scan } \\
\text { and read the article online }\end{array}$ & $\begin{array}{l}\text { ditation Mohammad Rahimi N, Mahdavinejad R, Attarzadeh Hosseini SR, Negahban H. Effect of Dynamic Neuromuscular } \\
\text { Stabilization Breathing Exercises on Some Spirometry Indices of Sedentary Students With Poor Posture. Physical Treatments. } \\
2019 ; 9(3): 169-176 . \text { http://dx.doi.org/10.32598/ptj.9.3.169 }\end{array}$ \\
doi $:$ : http://dx.doi.org/10.32598/ptj.9.3.169
\end{tabular}

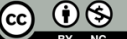

Article info:

Received: 05 Jan 2019

Accepted: 13 Apr 2019

Available Online: 01 Jul 2019

Keywords:

Dynamic neuromuscular stabilization, Breathing exercise, Respiratory function, Integrated spinal stabilizing system

\section{A B S T R A C T}

Purpose: Dynamic Neuromuscular Stabilization (DNS) approach is developed based on neurodevelopmental kinesiology and reflex-mediated core stabilization concepts. But the outcomes of this approach remain unclear. So changes in some spirometry indices in response to DNS breathing exercises in sedentary students with poor posture will be explored.

Methods: In this single-group pretest-posttest study design, we involved 26 male volunteer sedentary students with poor posture. First, a pretest of the spirometry indices (Maximum Voluntary Ventilation (MVV), Forced Expiratory Volume in first second (FEV1), Forced Vital Capacity (FVC), and FEV1/FVC ratio) were done each participant in random order. DNS breathing exercise protocol (six times a week, for six weeks) were trained and after completion of training, the post-test of the same parameters was performed. Descriptive statistical and the paired-sample test were used to analyze.

Results: The findings of data analysis indicated that the effect of DNS breathing exercise on respiratory function and significant improvements were observed in post-test compared with pre-test in the following parameters: MVV $(178.0 \pm 21.5$ vs $141.0 \pm 30.41 / \mathrm{min}, \mathrm{P}<0.001), \mathrm{FEV} 1$ (4.7 \pm 0.5 vs $4.0 \pm 0.5 \mathrm{~L}, \mathrm{P}<0.001)$, FVC (5.0 \pm 0.7 vs $4.4 \pm 0.6 \mathrm{~L}, \mathrm{P}<0.001)$, and $\mathrm{FEV} 1 / \mathrm{FVC}$ $(0.95 \pm 0.05$ vs $0.92 \pm 0.07 \mathrm{~L}, \mathrm{P}<0.001)$.

Conclusion: It can be concluded that DNS breathing exercise is an effective protocol to significantly improve respiratory function. Moreover, it can be deduced that DNS breathing exercise with a focus on the Integrated Spinal Stabilizing System (ISSS) and breathing techniques can serve as an effective instructive approach to prevent risks of malalignment.

\footnotetext{
* Corresponding Author:

Reza Mahdavinejad, PhD.

Address: Department of Sports Injuries and Corrective Exercises, Faculty of Sport Sciences, University of Isfahan, Isfahan, Iran

Phone: +98 (313) 7932573

E-mail: r.mahdavinejad@spr.ui.ac.ir
} 


\section{Highlights}

- The outcomes showed that the DNS breathing exercises based on developmental postural-locomotion models that utilized to stimulate ideal muscle coordination and define breathing stereotype significantly improved respiratory function.

\section{Plain Language Summary}

To improve the respiratory function of sedentary people with poor posture, researchers regularly investigate innovative and effective exercise methods and approaches. Also, when the breathing program frequently performed under numerous positions continuously, the respiratory training will be practical and becomes a choice program that this research is presenting one of the new procedures.

\section{Introduction}

he contemporary sedentary lifestyle leads  to increased functional disorders of the musculoskeletal system and poor posture [1]. Since the posture describes the mechanical impact of the sense of movement, as well as the balance and coordination of the musculoskeletal system [2], any blemish in the alignment can alter the function of numerous segments or specific organs as well [3]. So that even localized and temporary postural defects also have a significant influence on spirometry parameters [4].

Researchers reported that thoracic hyper kyphosis and malalignment of the chest significantly impaired the mechanics of the respiratory system, dwindle lung volumes [5], and alter the motor control strategy [6]. Additionally, respiratory malfunction that results from movement impairment of the rib cage and thoracic alignment [5], affects the mechanical efficiency of the respiratory muscles. Consequently, reduces the activation of these muscles especially the capability of diaphragm force generation [5]. Dimitriadis et al. (2013) reported that weakness of the flexor and extensor neck muscles and the upper back extensor muscles have negative effects on the inspiratory capability [7].

Recently, due to non-ergonomic working and studying conditions, sedentary lifestyles, and emotional and nervous tension in contemporary modern life and strongly linked with spinal impairment [8], having plans for the prevention of spinal alignment and respiratory function disorders should be regarded. Therefore, recognizing factors and actions to improve diaphragmatic efficiency is crucial. Multiple studies have shown that correct postural alignment is one of the most critical factors for optimal respiratory function $[3,4,9]$. Even temporary and rather minor postural disturbances have a significant impact on breathing function [3]. Also the function of the main respiratory muscle - the diaphragm is limited by abnormal posture, resulting in increased thoracic breathing pattern [10]. Scientific literature shows the effect of different core exercises [11], motor control exercises of the lumbar-pelvic region [12] and deep muscle training [3] on respiratory parameters and abdominal strength, improved respiratory function and the quality of posture and breathing, respectively. But, the initial alignment and motor function of the thorax is essential for physiologically balanced breathing and postural function [13]. Also, the mechanical action of the diaphragm and respiratory advantage depends on its relationship and anatomical alignment with the rib cage [14] that is not considered in previous studies.

Optimal trunk stabilization forms a cornerstone for all training programs [15]. It is critical to correct postural defects, restore the mobility of costovertebral joints, and to mobilize the intercostal tissue, and trunk fascia in breathing disorders treatment [16]. Stabilization and breathing patterns are closely interconnected, since breathing muscles serve as stabilizers and vice versa. Therefore it is convenient to train respiratorypostural function in integration and in various positions [16]. Dynamic Neuromuscular Stabilization (DNS) approach that is assessment and treatment approach based on developmental kinesiology models $[15,17,18]$ may serve such purpose.

The supported joints and all segments are brought into a functionally central position, at training precise muscle coordination by locating the subjects into different developmental positions is one of the most fundamental purposes of the DNS approach. Moreover, the combination of spinal stabilization and breathing pattern during daily activities is regarded $[12,13]$. 
If the breathing pattern is abnormal, then there will be no normal pattern of movement [14]. So taking part in rehabilitation schedules that affect respiratory muscles is crucial for the sedentary persons, notably those with poor posture, to deal with these postural, muscular and respiratory alterations. The aim of DNS breathing exercise - as a prerequisite for an integrated correction program - is to achieve the improvement of respiration and the coordination of local and global muscle complexes. Hence, this study was to evaluate the change in respiratory function in response to DNS breathing exercises in sedentary students with poor posture.

\section{Materials and Methods}

This single-group pretest-posttest study design was carried out on twenty-six poor posture sedentary male students (age: $20.6 \pm 1.2$ years; weight: $70.9 \pm 1.7 \mathrm{Kg}$; height $178.6 \pm 4.6 \mathrm{~cm}$, and BMI: $22.1 \pm 3.2 \mathrm{~kg} / \mathrm{m}^{2}$. Before the start of the study, all participants signed an informed consent form and they could give up the study at any time.

Inclusion criteria were consisted of: male gender, aged between 19-23 years old, BMI between 19-25 kg/ $\mathrm{m}^{2}$. being non-smoker and poor posture score between 11-15 points [15].

The distinct initial screening was according to the standardized method [15]. This practical method utilizes the following division of body posture: I. Perfect, II. Good, III. Poor and IV. Bad. This method was used as an inclusion criterion for the research. Postural alignment was evaluated by a qualified examiner and was visually done. The participant was examined in the habitual, relaxed posture that is usually adopted. Often, it takes some time for the participant to adopt the usual posture because of tenseness, uneasiness, or uncertainty. To limit individuals adjusting their position during evaluation, participants were said "You will be standing when the assessment is conducted; try to stand naturally. How do you feel now?"

In the standing positions in sagittal left view, the assessment is the same as the observation for head to pelvic scanning (head and neck posture, shoulder, chest, spin, pelvic) examinations each posture quality levels have five postural parts and is given point from 1-4 [15]. So a low score points out better body segment alignment. In this way, the classification of participants was based on the classification of postures including: Perfect posture (5 points), Good (almost perfect) posture (6-10 points), Poor posture (11-15 points), Bad posture (16-20 points).
Therefore, the students who have scored points 11-15 (poor posture) selected as subjects [15].

The assessment was visually performed by a qualified examiner between 8:00 am to $12: 00 \mathrm{pm}$, which we believed would minimize the influences of natural intraindividual postural changes that may occur during the day [16].

Exclusion criteria were including deformity of the chest, having background diseases (respiratory, neurologic, neuromuscular and cardiovascular), vestibular defects, former cardiac or thoracic surgery, and spinal postural alignment or upper and lower limb pathologies.

A standard survey was utilized to determine the activity level of volunteers. The survey, alike to the Persian translation of the Tegner activity scale, is scored from a high of 10 (competitive sports) to a low of 1 (sedentary) [17]. All participants were the sedentary level. The Persian-version of these instruments has acceptable psychometric properties for Iranian patients [18].

\section{Respiratory function test}

Before and after the training program, respiratory function was assessed via spirometry tests (Quark-Pulmonary, made in Italia). The parameters evaluated were Maximum Voluntary Ventilation (MVV), Forced Expiratory Volume in the first second (FEV1), Forced Vital Capacity (FVC), and FEV1/FVC, in standing position based on American Thoracic Society (ATS) and the European Respiratory Society (ERS) [19].

\section{Intervention}

The DNS breathing exercises performed six sessions a week ( 3 sessions of supervised exercise and 3 sessions of home-based exercise) for 6 weeks. First of all, Verbal signs, manual guide and visual feedback were given during the intervention to participants learned how to adjust the pelvis, spine, ribcage, scapula alignment and abdominal wall shape in various positions [20, 21]. Moreover, to motivate the participants to take part in practice sessions regularly, they were taught how the repetition of impaired movements, poor posture and sustained alignments may affect neuromusculoskeletal health. In the first week, all participants have personally trained the breathing exercise program (3 patterns).

Another week (2-6), the developmental stabilization patterns of a healthy infant based on the DNS approach done each exercise session per week (Table 1). Inhala- 
tion and exhalation were done respectively through the nose and the mouth. Also, during the breathing, the diaphragm falls into the abdominal cavity during the inhalation, the ribs rise and move laterally, expand the transverse dimensions of the thorax [20, 22-24]. The same DNS breathing exercise program was performed with participants at home. A book and a laminated card describing these exercises were given to the participants and encouraged to perform them daily at home. The compliance was reported by participants, daily.

Exercise intensity based on developmental positions automatically proper activation stereotype of stabilization and breathing of natural postural-locomotion patterns as defined by developmental kinesiology. This aim can be achieved via the activation of the stabilizers when placing the participants in developmental positions of an infant at third three months of developmental age to 13 months, as performed in this study [20, 22-25]. The protocol is implemented under the supervision of a trainer. The participants performed the exercises and the trainer corrected the performance if necessary. Besides, the participants represented their consent not to attend other courses or physical activities.

The statistical analysis was performed with statistical software namely SPSS version 16.0 (SPSS, Chicago, Illinois). All parameter outcomes were evaluated for each participant and then mean and standard deviation (Mean \pm SD) were computed by descriptive statistics test in pre and post session. Kolmogorov-Smirnov tests were utilized to examine the normality distribution. So to compare the mean between pre and posttest data, pairedsample t-test was used. Moreover, percentage change

Table 1. DNS breathing exercise protocol

\begin{tabular}{|c|c|c|}
\hline Week and Session & Exercise Description & Set \\
\hline \multirow{3}{*}{$\begin{array}{l}\text { Week } 1 \\
\text { Session 1-6 }\end{array}$} & Supine breathing exercise & \\
\hline & Prone breathing exercise & \\
\hline & breathing exercise $90 / 90$ & \\
\hline \multirow{5}{*}{$\begin{array}{c}\text { Week } 2 \\
\text { Session } 7-12\end{array}$} & Prone position: elbow support (3 months old position) & \\
\hline & Supine position 90/90: arm outside the body ( 3 months old position) & \\
\hline & Supine position 90/90: hand on the abdomen (4 months old position) & \\
\hline & Creeping position (one hip and knee in flexion): elbow support, ASIS and medial epicon- & \\
\hline & dyle of the opposite knee ( 4.5 months old position) & Set 1: \\
\hline \multirow{5}{*}{$\begin{array}{c}\text { Week } 3 \\
\text { Session } 13-18\end{array}$} & Rolling pattern (ipsilateral) position (5 months old position) & 10 repetitions \\
\hline & Supine position 90/90: hand on the knee (5 months old position) & \multirow[t]{2}{*}{1 second inhale: 2 second exhale } \\
\hline & Prone position: hand and knee support (elbow is extension) (6 months old position) & \\
\hline & Supine position (hip and knee in 45-degree flexion): hand on the foot (6 months old position) & $60-90$ second rest period \\
\hline & & Set 2: \\
\hline \multirow{5}{*}{$\begin{array}{c}\text { Week } 4 \\
\text { Session } 19-24\end{array}$} & Quadruped position (the angle between trunk and hip is 120 degree) (7 months old position) & 15 repetitions \\
\hline & Quadruped position (the angle between trunk and hip is 90 degree) (7 months old position) & \multirow{2}{*}{2 second inhale: 4 second exhale } \\
\hline & Oblique sit position (side plank) with arm and lateral knee support (7 months old position) & \\
\hline & oblique-sitting position with hand support(elbow is extended)( 8 months old position) & $60-90$ second rest period \\
\hline & & Set 3: \\
\hline \multirow{4}{*}{$\begin{array}{c}\text { Week } 5 \\
\text { Session } 25-30\end{array}$} & Crawling position (9 months old position) & \multirow{4}{*}{$\begin{array}{c}3 \text { second inhale: } 6 \text { second exhale } \\
120-150 \text { second rest period }\end{array}$} \\
\hline & Sitting position (Keep the spine upright and elongated) (10 months old position) & \\
\hline & Side-lying (side plank) with hand, lateral knee and opposite foot support (10 months old position) & \\
\hline & $\begin{array}{l}\text { Raising position (Keep the spine forward and elongated and one leg kneeling) (11 months old } \\
\text { position) }\end{array}$ & \\
\hline \multirow{5}{*}{$\begin{array}{c}\text { Week } 6 \\
\text { Session } 31-36\end{array}$} & High kneeling" position (Keep the spine upright and elongated and one leg kneeling) (11 & \\
\hline & months old position) & \\
\hline & Bear position (12 months old position) & \\
\hline & Squat position (12 months old position) & \\
\hline & Standing position (initial standing position) (13 months old position) & \\
\hline
\end{tabular}


was calculated as $(($ posttest-pretest $) /$ pretest $) \times 100)$ and the effect size computed with G Power 3.1 software [26]. The significance level was established at $\mathrm{P}<0.05$.

\section{Results}

According to the findings of study (Table 2), after 6-week there was a significant difference between the mean of the pre- and post-test of spirometry parameters including MVV, FEV1, FVC, and FEV1/FVC ratio.

\section{Discussion}

According to the results of this study, six weeks of DNS breathing exercises as a non-invasive method with emphasis on the integrated spinal stabilizing system (ISSS) [22, 27], precise muscle timing and coordination to have movement efficiency and breathing technique $[12,20,22]$ shown statistically and clinically significant improvement in the spirometry values (MVV, FVC, FEV1, FEV1/FVC ratio).

Among respiratory maneuvers, MVV is more likely to be considered as a dynamic ventilation test because the reduction of MVV in neuromuscular and cardiovascular diseases, as well as those suffering from airway stenosis, is obvious. The increase in MVV from $141 \pm 30.48$ to $178 \pm 21.53(26.24 \%$ increased, $\mathrm{P}<0.001)$ was statistically and clinically significant. Similar results were reported in Bezzoli et al. (2016) who examined the effect of lumbo-pelvic motor control exercises on pulmonary function in obese individuals [28]. Increasing MVV is associated with low airway resistance and enhanced respiratory muscle strength and coordination [29]. However, the present study indicates probably developed respiratory muscle strength and coordination and better oxygen availability lead to increase the MVV.
The incremental changes in the two factors of FVC, FEV1 were $12.61 \%$ and $16 / 42 \%$, respectively. In addition, the effect size of the exercises in two factors was 0.81 and 1.11, indicating a change of approximately four-fifths the baseline standard deviation considered as large [30]. These changes can be related to neuromuscular coordination and the more activity and desirable of the diaphragm muscle as the main respiratory muscle that plays a stability role as well as other respiratory muscles [31, 32].

Positive effects in the FVC may also show alterations in lung compliance [33]. Malatova and Drevikovska (2009) demonstrated that weakness and poor coordination of the diaphragm may lead to lumbar instability and impairment of its movement patterns. Conversely, loss of lumbar region stability due to poor posture and improper direction has a negative effect on the function and efficiency of respiration [34].

The results were consistent with the findings of Bezzoli et al. (2016) that examined the effects of pelvic lung motor control exercises on pulmonary function in obese people [28]. Joshi et al. (1992) and Dinesh et al. (2018) also found that pranayama exercises improve respiratory function by increasing FEV and FEV1 [31, 35]. Sivakumar et al. (2011) also investigated the effect of deep interpretation exercises on lung function, which was consistent with the results of the present study (FVC) [33]. Moreover, similar and comparable results have been reported by Obayashi and colleagues (2012) that confirms the effect of breathing muscle exercise on ameliorating respiratory function [36]. FVC and FEV1, are included of the most critical pulmonary function maneuvers.

An obstruction in the respiratory airways or weakness and imbalance between the respiratory muscles (diaphragm, intercostal muscles, and abdominal muscles)

Table 2. The changes of parameters from baseline to 6 weeks

\begin{tabular}{|c|c|c|c|c|c|}
\hline \multirow{2}{*}{ Parameters } & \multicolumn{2}{|c|}{ Means $\pm S D$} & \multirow{2}{*}{ Changes (\%) } & \multirow{2}{*}{ ES } & \multirow{2}{*}{$\mathbf{P}^{*}$} \\
\hline & Pre-test & Post-test & & & \\
\hline $\mathrm{MVV}$ (L/min) & $141.0 \pm 30.4$ & $178.0 \pm 21.5$ & 26.2 & 1.32 & $<0.001$ \\
\hline FEV1 (L) & $4.0 \pm 0.5$ & $4.7 \pm 0.5$ & 16.4 & 1.11 & $<0.001$ \\
\hline FVC (L) & $4.4 \pm 0.6$ & $5.0 \pm 0.7$ & 12.6 & 0.81 & $<0.001$ \\
\hline FEV1/FVC (L) & $0.92 \pm 0.07$ & $0.95 \pm 0.05$ & 3.2 & 0.49 & $<0.001$ \\
\hline
\end{tabular}

MVV: Maximum Voluntary Ventilation; FEV1: Forced Expiratory Volume in first second; FVC: Forced Vital Capacity 
alter the values of FVC and FEV1. The lumbar-pelvic deep cylinder including the diaphragm, transverse abdominal, and pelvic floor muscles, modulate the intraabdominal pressure regarded as an important requirement to provide stability and respiration. Abdominal tension also rais es the diaphragm inspiration performance on the ribs through two components Insertional and oppositional force [37].

Increased residual volume is a result of expiratory muscle deficiency, as well as neuromuscular insufficiency, along with diminished lung elasticity and limited FVC levels [32]. Before participating in the DNS breathing exercises program the mean FEV1/FVC ratio was reported 0.92 liters, which indicates the optimal ventilation function of the participants [32]. However, the incremental changes in this ratio after the exercise period increased by $26.3 \%$ and the effect size of 0.49 (average effect size) was statistically significant $(\mathrm{P}<0.05)$.

These results were consistent with the findings of Bezzoli et al. (2016), Kondam et al. (2015), Kumar et al. (2018) [28, 38, 39]. Bezzoli et al., Who performed the effects of motor control exercises on the respiratory function of the lumbar muscles, as one of the defective factors in sedentary and obese people, reported significant enhancement of respiratory function factors in the experimental group [28]. Motor control exercises can be significantly used as a strategy that uses the central nervous syste $m$ to control and coordinate the muscle contraction of all muscle groups involved [40]. This process is dependent on sensory systems and motor components, including muscles, ligaments, joints, and fascias, as well as neuro-cognitive processes [28]. Kondam et al. Confirmed the effect of pranayama exercises on the FEV1/FVC ratio by examining the effect of yoga exercises (pranayama and suryanamaskar) on the pulmonary function of a young person in a 6-month period [38]. In addition, the Kumar et al. study, which was performed on 55 medical students, showed a significant effect of yoga exercises on pulmonary function [39]. This seems to be achieved by increasing and improving the maximum expiratory pressure that results from the upgraded strength of expiratory muscles. Moreover, in the second stage ameliorating these factors can be as a result of promoting the coordination of respiratory muscles and the alignment of the spine and chest. The normal and neutral position of the spine facilitates the function of exhaled muscles (transverse abdomen, intercostal muscles, pelvic floor muscles, and external oblique).

DNS is a functional approach view that integrates brain stimulation with manipulation, mobilization, pos- tural aw areness, breathing training and education to achieve optimal, global body function $[33,34]$. Moreover, c a n be regarded as a "neutral" and "optimal" alignment of the head and neck, spinal, thorax, and pelvis and strongly recommends that a healthy sensorimotor system is required to design an optimal function that sets the joints in centration $[22,23]$. The outcome of joint centration is minimal mechanical stress on relevant passive tissues and dwindles the over activation of superficial muscles [22].

The protocol also discusses the spirometric values. To educate the participants about the integration of a proper pattern of breathing and alignment within the daily living activities is one of the main goals of DNS breathing exercises. Our study proves confident respiratory results can be taken by appointing DNS breathing exercises. It is suggested that clinically it is crucial to regard DNS breathing exercises based on ideal ontogenetic patterns may be utilized to accomplish optimal for student's subjects especially when the improvement influence is proposing to develop with weakened respiratory function.

\section{Ethical Considerations}

\section{Compliance with ethical guidelines}

Permiss ion was taken from the Ethics Committee of the Isfahan University, Isfahan, Iran (Code: IR.UI. REC.1398.006), and was carried out according to the Helsinki Protocol.

\section{Funding}

This work was financially supported by Isfahan University.

\section{Authors' contributions}

All authors contributed in designing, running, and writing all parts of the research.

\section{Conflict of interest}

The authors declared no conflict of interest.

\section{Acknowledgments}

Authors wish to thank Isfahan University for financial support (Ph.D thesis grant). Also, we thank the participants in the study. 


\section{References}

[1] Novotná NB, Slováková M. The current problem of school children-lack of physical activity. European Researcher 2016; 3(4):231-8. [DOI:10.13187/er.2016.105.231]

[2] Latalski M, Bylina J, Fatyga M, Repko M, Filipovic M, Jarosz MJ, Borowicz KB, Matuszewski L, Trzpis T, et al. Risk factors of postural defects in children at school age. Annals of Agricultural and Environmental Medicine. 2013; 20(3):583-7.

[3] Szczygieł E, Blaut J, Zielonka-Pycka K, Tomaszewski K, Golec J, Czechowska D, et al. The impact of deep muscle training on the quality of posture and breathing. Journal of Motor Behavior. 2018; 50(2):219-27. [DOI:10.1080/00222895. 2017.1327413] [PMID]

[4] Pawlicka-Lisowska A, Motylewski S, Lisowski J, Michalak K, Poziomska-Piatkowska E. Faulty posture and selected respiratory indicators. Polski Merkuriusz Lekarski. 2013; 35(206):67-71. [PMID]

[5] Dajah S Al, Muthusamy H. Effects of back and respiratory muscle exercises on posture and respiratory function in elderly patients with osteoporosis. International Journal of Therapy and Rehabilitation. 2015; 22(5):233-7. [DOI:10.12968/iitr.2015.22.5.233]

[6] Bradley H, Esformes JD. Breathing pattern disorders and functional movement. International Journal of Sports Physical Therapy. 2014; 9(1):28-39. [PMID] [PMCID]

[7] Dimitriadis Z, Kapreli E, Strimpakos N OJ. Respiratory weakness in patients with chronic neck pain. Manual Therapy. 2013; 18(3):248-53. [DOI:10.1016/j.math.2012.10.014] [PMID]

[8] O'Sullivan PB, Dankaerts W, Burnett AF, Farrell GT, Jefford E, Naylor CS, et al. Effect of different upright sitting postures on spinal-pelvic curvature and trunk muscle activation in a pain-free population. Spine. 2006; 31(19):707-12. [DOI:10.1097/01.brs.0000234735.98075.50] [PMID]

[9] Kim MS, Cha YJ, Choi JD. Correlation between forward head posture, respiratory functions, and respiratory accessory muscles in young adults. Journal of Back and Musculoskeletal Rehabilitation. 2017; 30(4):711-5. [DOI:10.3233/ BMR-140253] [PMID]

[10] Hodges PW, Heijnen I, Gandevia SC. Postural activity of the diaphragm is reduced in humans when respiratory demand increases. The Journal of Physiology. 2001; 537(3):9991008. [DOI:10.1113/jphysiol.2001.012648] [PMID] [PMCID]

[11] Cavaggioni L, Ongaro L, Zannin E, Iaia FM, Alberti G. Effects of different core exercises on respiratory parameters and abdominal strength. The Journal of Physical Therapy Science. 2015; 27(10):3249-53. [DOI:10.1589/jpts.27.3249] [PMID] [PMCID]

[12] Bezzoli E, Andreotti D, Pianta L, Mascheroni M, Piccinno L, Puricelli L, et al. Motor control exercises of the lumbarpelvic region improve respiratory function in obese men. A pilot study. Disability and Rehabilitation. 2018; 40(2):152-8. [DOI:10.1016/j.math.2016.05.131]

[13] Chaitow L, Gilbert C, Morrison D. Recognizing and treating breathing disorders e-book: a multidisciplinary approach. Edinburgh: Elsevier Health Sciences; 2014.
[14] Mead J. Functional significance of the area of apposition of diaphragm to rib cage. American Review of Respiratory Disease. 1979; 119(2p2):31-2.

[15] Frank C, Kobesova A, Kolar P. Dynamic neuromuscular stabilization \& sports rehabilitation. International Journal of Sports Physical Therapy. 2013; 8(1):62-73. [PMID] [PMCID]

[16] Page P, Frank C, Lardner R. Assessment and treatment of muscle imbalance: The Janda approach. Journal of Orthopedic \& Sports Physical Therapy. 2011; 41(10):799-800. [PMCID]

[17] Kobesova A, Kolar P. Developmental kinesiology: Three levels of motor control in the assessment and treatment of the motor system. Journal of Bodywork and Movement Therapies. 2014; 18(1):23-33. [DOI:10.1016/j.jbmt.2013.04.002] [PMID]

[18] Kolar P, Sulc J, Kyncl M, Sanda J, Neuwirth J, Bokarius A $\mathrm{V}$, et al. Stabilizing function of the diaphragm: dynamic MRI and synchronized spirometric assessment. Journal of Applied Physiology. 2010; 109(4):1064-71. [DOI:10.1152/japplphysiol.01216.2009] [PMID]

[19] Kolar P, Kobesova A. Postural-locomotion function in the diagnosis and treatment of movement disorders. Clinical Chiropractic. 2010; 1(13):58-68. [DOI:10.1016/j. clch.2010.02.063]

[20] Oh DS, Park SE. The effect of lumbar stabilization exercise on the pulmonary function of stroke patients. The Journal of Physical Therapy Science. 2016; 28(6):1896-900. [DOI:10.1589/jpts.28.1896] [PMID] [PMCID]

[21] Lewit K. Relation of faulty respiration to posture, with clinical implications. The Journal of the American Osteopathic Association. 1980; 79(8):525-9. [PMID]

[22] Bendíková E, Görner K, Paugshová B. Exercise programme for schoolgirl with poor posture. Journal of Education, Health and Sport. 2016; 6(13):54-64. [DOI: 10.5281/zenodo.232974]

[23] Canales JZ, Fiquer JT, Campos RN, Soeiro-de-Souza MG, Moreno RA. Investigation of associations between recurrence of major depressive disorder and spinal posture alignment: A quantitative cross-sectional study. Gait \& Posture. 2017; 52:258-64. [DOI:10.1016/j.gaitpost.2016.12.011] [PMID]

[24] Kiesel K, Rhodes T, Mueller J, Waninger A, Butler R. Development of a screening protocol to identify individuals with dysfunctional breathing. International Journal of Sports Physical Therapy. 2017; 12(5):774-86. [DOI:10.26603/ ijspt20170774] [PMID] [PMCID]

[25] Negahban H, Mostafaee N, Sohani SM, Mazaheri M, Goharpey S, Salavati M, et al. Reliability and validity of the Tegner and Marx activity rating scales in Iranian patients with anterior cruciate ligament injury. Disability and Rehabilitation. 2011; 33(22-23):2305-10. [DOI:10.3109/09638288.2011.5 70409] [PMID]

[26] Miller MR, Hankinson J, Brusasco V, Burgos F, Casaburi R, Coates A, et al. Standardisation of spirometry. European Respiratory Journal. 2005; 26(2):319-38. [DOI:10.1183/09031 936.05.00034805] [PMID]

[27] Lim YL, Lepsikova M, Singh DK. Effects of dynamic neuromuscular stabilization on lumbar flexion kinematics and 
posture among adults with chronic non-specific low back pain: A study protocol. Berlin: Springer; 2016.

[28] Kobesova A, Dzvonik J, Kolar P, Sardina A, Andel R. Effects of shoulder girdle dynamic stabilization exercise on hand muscle strength. Isokinetics and Exercise Science. 2015; 23(1):21-32. [DOI:10.3233/IES-140560]

[29] Gupta M, Jain S, Kumar V. Effect of Raja Yoga Meditation on pulmonary functions of young obese medical students. Prof. RK Sharma. 2016; 7(2):1-8. [DOI:10.5958/09765506.2016.00054.1]

[30] Husted JA, Cook RJ, Farewell VT, Gladman DD. Methods for assessing responsiveness: a critical review and recommendations. Journal of Clinical Epidemiology. 2000; 53(5):459-68. [DOI:10.1016/S0895-4356(99)00206-1]

[31] Shirley D, Hodges PW, Eriksson AEM, Gandevia SC. Spinal stiffness changes throughout the respiratory cycle. Journal of Applied Physiology. 2003; 95(4):1467-75. [DOI:10.1152/ japplphysiol.00939.2002] [PMID]

[32] Lakhera SC, Mathew L, Rastogi SK, Sen JG. Pulmonary function of Indian athletes and sportsmen: comparison with American athletes. Indian Journal of Physiology and Pharmacology. 1984; 28(3):187-94. [PMID]

[33] Cohen J. Statistical power analysis for the behavioral sciences. Abingdon: Academic Press; 1969.

[34] Dinesh T, Rajajeyakumar M, Sakila S. Effect of 12 weeks of slow breathing exercise practice on anthropometric parameters in healthy volunteers. National Journal of Physiology, Pharmacy and Pharmacology. 2018; 8(12):1650-3.

[35] Attarzadeh Hoeini SR, Hojati Oshtovani Z, Soltani H, Hossein Kakhk SA. [Changes in pulmonary function and peak oxygen consumption in response to interval aerobic training in sedentary girls (Persian)]. Quarterly Journal of Sabzevar University of Medical Sciences. 2012; 19(1):42-51.

[36] Sivakumar G, Prabhu K, Baliga R, Pai MK, Manjunatha S. Acute effects of deep breathing for a short duration (2-10 minutes) on pulmonary functions in healthy young volunteers. Indian Journal of Physiology and Pharmacology. 2011; 55(2):154-9. [PMID]

[37] Malatova R, Dřevikovská P. Testing procedures for abdominal muscles using the muscle dynamometer SD02. Proceedings of the Institution of Mechanical Engineers. 2009; 223(8):1041-8. [DOI:10.1243/09544119JEIM575] [PMID]

[38] Kondam A, Chandrasekhar M, Punita P, Varadharaju B, Suresh M, Karthik S. Combined effects of pranayama and suryanamaskar on dynamic spirometric values in normal young subjects. National Journal of Physiology, Pharmacy and Pharmacology. 2015; 5(2):79-88. [DOI:10.5455/ njppp.2015.5.020920141]

[39] Kumar D. Role of regular yoga practice in improvement of various pulmonary parameters in first year medical students. International Journal of Research in Medical Sciences. 2018; 6(7):2523-7. [DOI:10.18203/2320-6012.jirms20182848]

[40] De Troyer A, Boriek AM. Mechanics of the respiratory muscles. Comprehensive Physiology. 2011; 1(3):1273-300. [DOI:10.1002/cphy.c100009] [PMCID] 